\title{
On the Location of Multiple Failure Slip Surfaces in Slope Stability Problems Using the Meshless SPH Algorithm
}

\author{
Liang Li $\left({ }^{1},{ }^{1}\right.$ Ming Zhai, ${ }^{1}$ Xianzhang Ling $\left(\mathbb{D},{ }^{1}\right.$ Xuesong Chu, ${ }^{1}$ Bin $\mathrm{Hu}\left(\mathbb{D},{ }^{2}\right.$ \\ and Yungming Cheng ${ }^{1}$ \\ ${ }^{1}$ School of Civil Engineering, Qingdao University of Technology, Qingdao, China \\ ${ }^{2}$ School of Resources and Environmental Engineering, Wuhan University of Science and Technology, Wuhan, China \\ Correspondence should be addressed to Liang Li; liliang@qut.edu.cn, Xianzhang Ling; lingxianzhang@qut.edu.cn, and Bin Hu; \\ hbin74@wust.edu.cn
}

Received 24 January 2020; Revised 29 April 2020; Accepted 9 June 2020; Published 30 June 2020

Academic Editor: Fan Gu

Copyright (C) 2020 Liang Li et al. This is an open access article distributed under the Creative Commons Attribution License, which permits unrestricted use, distribution, and reproduction in any medium, provided the original work is properly cited.

The occurrence of multiple critical slip surfaces with equal importance in rehabilitating and reinforcing slopes has been frequently observed in geotechnical engineering practices. The simultaneous determination of these potential slip surfaces is, however, not trivial. This paper presents a methodology based on the smoothed particle hydrodynamics (SPH) approach, which can simultaneously determine multiple failure slip surfaces and the debris flow process without previous knowledge or trial-and-error processes, and this methodology is validated against a slope with the presence of multiple critical slip surfaces. The proposed methodology serves as an efficient and effective alternative approach to traditional approaches, which involve cumbersome treatments performed by engineers based on their subjective experiences. The multiple sources of failure slip surfaces in slope stability are equivalent to multiple sources of initiation of slope failure, and it is found that SPH can provide a direct and systematic tool for identifying multiple failure slip surfaces. However, some minor potential problems are also found with the use of the SPH method in actual applications.

\section{Introduction}

The limit equilibrium method (LEM), finite element method, and finite difference-based strength reduction techniques are among the more conventional tools to determine the factor of safety (FS) at the critical slip surface in slope stability problems [1-18]; [19]; Li and Chu [20]. As a special form of limit analysis, the discontinuity layout optimization method (DLO) has recently been applied to slope stability problems by Krabbenhoft et al. [21], Smith and Gilbert [22], and Cheng [23]; Cheng [23] found various limitations to DLO, which Cheng [23] believed to be the inadequacies of the numerical implementation instead of fundamental problems related to DLO. For cases where multiple critical slip surfaces exist (same FS for different critical slip surfaces) and for those cases where the FS values of the local minima are only slightly higher than the critical value, these multiple critical slip surfaces are usually not considered in a systematic manner. However, for deposited embankments, tailing dams, or excavated slopes with complicated stratigraphy, the occurrence of several critical failure slip surfaces with slightly different or even equal FS values is not surprising, and such a condition should be properly considered in rehabilitating or reinforcing slopes. To properly address this issue, Cheng et al. [10] proposed a procedure to locate most of the dangerous failure slip surfaces by varying the search domains for the entrance and exit points. In the program SLOPE2000 by Cheng, multiple critical slip surfaces can be determined visually by the locations of those slip surfaces with an FS 2-5\% (or other values) higher than the global minimum FS. More precise locations of the other critical solutions can then be determined by changing the search domain for the entrance and exit points range. If there are several local minima in the solution with virtually the same FS, it is not easy to determine multiple critical slip surfaces from the visual observation in SLOPE2000. Reale et al. [24] developed a holistic multimodal optimization technique to locate and 
converge to multiple failure slip surfaces simultaneously. $\mathrm{Li}$ and Chu [25] used a correlation coefficient-based approach to find the multiple failure slip surfaces of interest from a large number of potential failure slip surfaces. There is no rigorous rule for determining the values of the algorithm parameters in Reale's approach [24], and the variation in their values may lead to the divergence of the optimization techniques. The calculations of the correlation coefficients among failure slip surfaces are time consuming in $\mathrm{Li}$ and Chu's approach [25]. It appears that the approach in SLOPE2000 by Cheng is the simplest method at present for normal problems, as no additional computation is required to determine the other critical slip surfaces. Determining the multiple failure slip surfaces for general cases in a systematic, automatic, and direct manner remains an open question.

This study aims to develop an alternative approach that combines a smoothed particle hydrodynamics (SPH) algorithm with strength reduction techniques to automatically locate the multiple failure slip surfaces for slopes with complex deposition layers. As a mesh-free method which can tolerate very large movement and distortion, SPH can simulate the whole process of slope failure, including the initiation, propagation, and end of the slope failure. A strength reduction technique serves as a tool to bring the slope from a stable state to the failure state.

The paper starts with a brief introduction to $\mathrm{SPH}$, followed by overviews of FEM- and FDM-based strength reduction techniques used to perform slope stability and to locate the multiple failure slip surfaces in LEM. Then, the proposed SPH methodology based on a self-developed program is used to simulate the whole process of slope failure to capture multiple failure slip surfaces. Finally, discussions and conclusions are given to provide insights into this interesting multiple critical solutions problem.

\section{Brief Review of Smoothed Particle Hydrodynamics (SPH)}

Smoothed particle hydrodynamics belongs to the category of meshless methods originally proposed by Lucy [26] and Gingold and Monaghan [27] for astrophysics problems. The strain and strain rates are large in SPH, and hence the use of frame-invariant Eulerian Jaumann stress rate is commonly adopted in the formulation. This method is actually a meshfree nonlocal Lagrangian method (where the coordinates move with the fluid), and the resolution of the method can be easily adjusted with respect to the various control variables. SPH discretizes the problem domains into a finite number of particles, embodying a set of physical parameters, such as mass, velocity, strain, and stress. One particle is connected with other particles within its support domain by a kernel function. The particle has a spatial distance over which the properties are "smoothed" by the specified kernel function. The physical quantities of any particle can be obtained by summing the relevant properties of all the particles that lie within the range of the kernel. The contributions of each particle to a property are weighted according to their distance from the particle of interest and their density. At the initiation of SPH simulation, all the particles move according to the external forces acting on them, and then those particles near the slope boundaries are hindered due to the counterforces from the slope boundaries, thereby yielding strains and stresses for these particles. Owing to the particle interaction, the strains and stresses will appear gradually in all the other particles. Elastic and plastic strains develop in accordance with the prescribed constitutive models. The particles will keep moving until the steady state is reached. Various researchers have contributed to the development of SPH over the last 30 years, and many different techniques have been proposed to overcome the basic limitations in the applications of SPH. Currently, SPH is applied in hydrodynamic, mechanical, blasting, dynamic impact, and geotechnical applications.

SPH has also been widely used in many research fields such as fluid mechanics [28-30] and geotechnical engineering [31-42] since the constitutive model was first implemented in SPH by Bui et al. [43]. There are some applications of SPH in slope stability problems, most notably the works by Ono (2010), Bui et al. [31, 32, 43], and Nonoyama et al. [35, 36]. For the final on-site measured failure profile and the run-off for the Shum Wan Road landslide in Hong Kong (failure in limonite and kaolinite), the measured results match relatively well with the SPH results by Wong [44], and the results are also better than those obtained by Chen and Lee [45] using FEM. The better results from SPH have inspired the authors to further investigate the application of meshless methods in slope stability analysis. An in-house Fortran-based SPH software package has been developed and has been validated through comparison with SPH and FEM results as well as physical model test results reported in the literature [38]. This software package is used to model the slope failure process.

\section{Slope Stability Analysis by Traditional Methods}

An example with a multiple critical slip surfaces problem by Cheng et al. [10] and Reale et al. [24] is reanalysed in this study. The slope has a height of $17 \mathrm{~m}$ and a series of gradient changes throughout the profile. As shown in Figure 1, the uniform soil parameters are cohesion $c=5 \mathrm{kPa}$ and frictional angle $\varphi=30^{\circ}$, while the unit weight is $20 \mathrm{kN} / \mathrm{m}^{3}$. Before this slope is reinforced or rehabilitated, the FS, especially of the failure slip surfaces, must be located. In this section, FEM and FDM are considered using a strength reduction technique in commercial numerical tools (e.g., Abaqus, FLAC, and Plaxis) to evaluate the FS and automatically locate the failure slip surface. In Abaqus and FLAC, the convergence study of mesh fineness on the calculation result is first investigated to ensure the resolution of the calculated results. The dilatancy angle $\psi$ is set to zero, and therefore, the nonassociated flow rule is adopted in FEM and FDM. In addition, the Spencer method with a noncircular slip surface is also applied using SLOPE2000 to determine the minimum FS and the multiple critical slip surfaces. The details of model parameters in FEM, FDM, and LEM are summarized in Table 1. The calculation results for FEM, FDM, and LEM are shown in Figures 2-4. The FS value obtained by FEM and 
FDM is 1.33, and LEM yields a comparable value of 1.33. The critical FS and slip surfaces from Abaqus, FLAC, and SLOPE2000 are similar to each other, and such a result is not surprising, as has been discussed by Cheng et al. [10]. The failure slip surfaces that are automatically identified by the strength reduction technique in Abaqus have not fully extended to form continuous profiles, but they indicate the presence of three sources of potential failure slip surfaces at different elevations, as shown in Figure 2. FDM in FLAC or Plaxis, however, provides only one full slip surface at the middle part of the slope. As discussed by Cheng et al. [10], there are various minor limitations in different FEM-based SRM programs, and FEM may not be able to give good or satisfactory results for all cases. Cheng et al. [10] discussed the difficulties of many commercial programs in generating multiple critical slip surfaces automatically during the analysis, and the limited trial tests of the updated commercial programs by the authors still reveal such limitations. The automatic evaluation of multiple critical slip surfaces using SRM still appears to be nontrivial.

For the present problem, LEM using SLOPE2000 can also provide multiple slip surfaces (local minima) by changing the entrance and exit ranges manually for different optimization searches after viewing the "trial surfaces range" in the program, but such a semiautomatic approach can be difficult if the locations of the multiple critical slip surfaces are near each other. The limitations of the classical methods are clearly illustrated through this example. For general cases, the presence of multiple critical slip surfaces is unknown to the engineers, and the reliance of commercial SRM programs, engineering experience, or trial-and-error processes may not be adequate. The authors would like to develop the tool for which multiple critical slip surfaces that are full slip surfaces can be determined without human interpretation or intervention, and this is the main purpose of the present work.

\section{Case Study Using an SPH-Based Approach}

As shown in Figure 5, the proposed SPH-based approach starts with the configuration of particle size and SPH-related parameters, and the stress and deformation for all the particles for the stable slope are then obtained by SPH, followed by strength reduction to decrease the strength of the soil. The soil particles will move, deform, yield, and slide accordingly. The failure slip surfaces can then be identified by analysing the contour plot of the deformations for all the particles at different times during the SPH simulation.

The multibenched slope in Figure 1 is revisited, and the whole process of slope failure is simulated using SPH. First, the slope domain is divided into 29365 circular particles, each of which has a radius of $0.1 \mathrm{~m}$. A time step $\Delta t$ of $0.0002 \mathrm{~s}$ and $h$ of $0.24 \mathrm{~m}$ based on the recommendation by Mao et al. [37] are adopted in the present analysis. The deformation of the slope particles after applying the gravity forces is shown in Figure 6, and only very slight deformations are found, indicating that the slope is at the stable state. Based on the FS values (approximately 1.35) obtained by classical methods, a slightly larger reduction factor (or FS) of 1.40 is used to

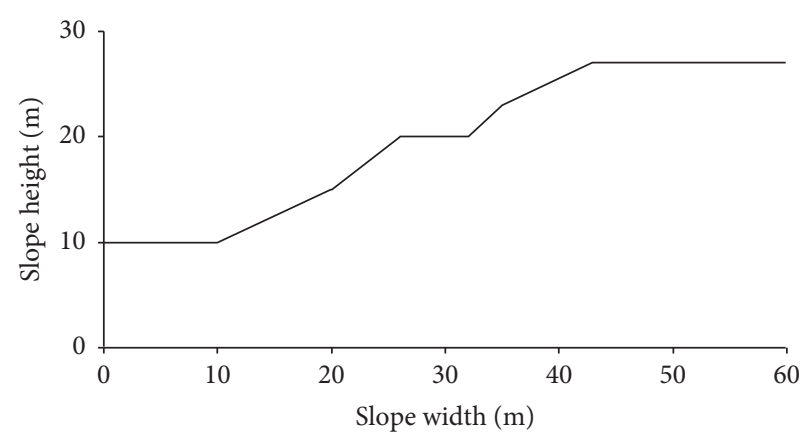

Figure 1: Geometry for multistage slope.

TABLE 1: Summary of model parameters in FEM, FDM, and LEM.

\begin{tabular}{lcccccc}
\hline Method & $\begin{array}{c}\gamma \\
\left(\mathrm{kN} / \mathrm{m}^{3}\right)\end{array}$ & $\begin{array}{c}C \\
(\mathrm{kPa})\end{array}$ & $\begin{array}{c}\varphi \\
(\text { degree })\end{array}$ & $E(\mathrm{kPa})$ & $\mu$ & $\begin{array}{c}\delta \\
(\text { degree })\end{array}$ \\
\hline LEM & 20 & 5 & 30 & NA & NA & NA \\
FEM & 20 & 5 & 30 & 100000 & 0.3 & 0 \\
FDM & 20 & 5 & 30 & 100000 & 0.3 & 0 \\
\hline
\end{tabular}

Note. $\gamma=$ unit weight, $c=$ cohesion, $\varphi=$ internal friction angle, $E=$ elastic modulus, $\mu=$ Poisson ratio, $\delta=$ dilation angle, and NA $=$ not applicable.

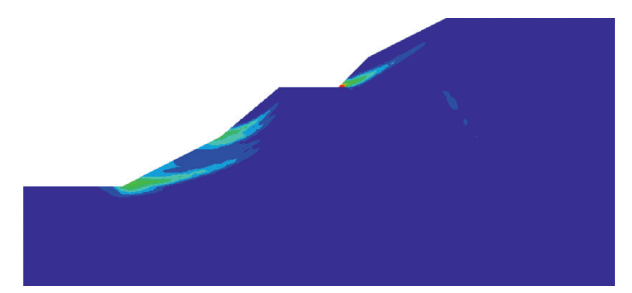

FIgURE 2: The contour plot of plastic strain magnitude in Abaqus with $\mathrm{FS}=1.33$.

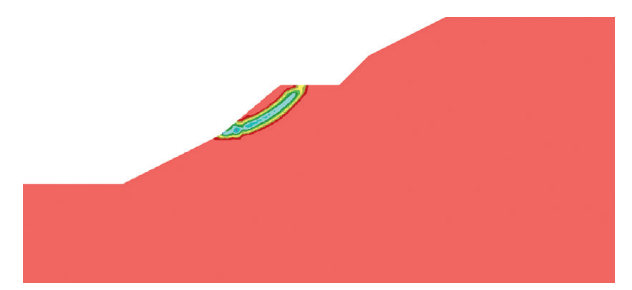

FIgURE 3: The contour plot of incremental plastic strain magnitude in FLAC with FS $=1.33$ (similar to Plaxis).

reduce the soil parameters in the $\mathrm{SPH}$ analysis. The reduced cohesion is $5 / 1.4$, and the tan value of reduced friction angle is $\tan (\pi / 6) / 1.4$; these parameters are defined in the SPH analysis to simulate the slope failure process. The slope has reached a steady state at $t=6 \mathrm{~s}$, and the plots of the contour of slope deformation at different times representing the initiation, propagation, and the final steady state of slope failure are shown in Figure 7. Figure 7(a) shows the contour of deformation at $t=1 \mathrm{~s}$, and it is clear that two failure zones have been initialized, including a smaller one in the middle of the slope and a larger one ranging from the top of the 


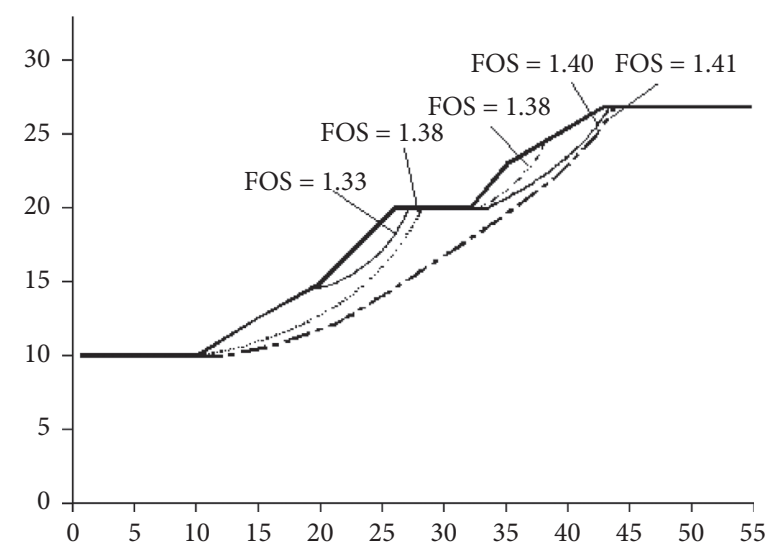

Figure 4: The multiple failure slip surfaces with minimum $F S=1.33$ in LEM.

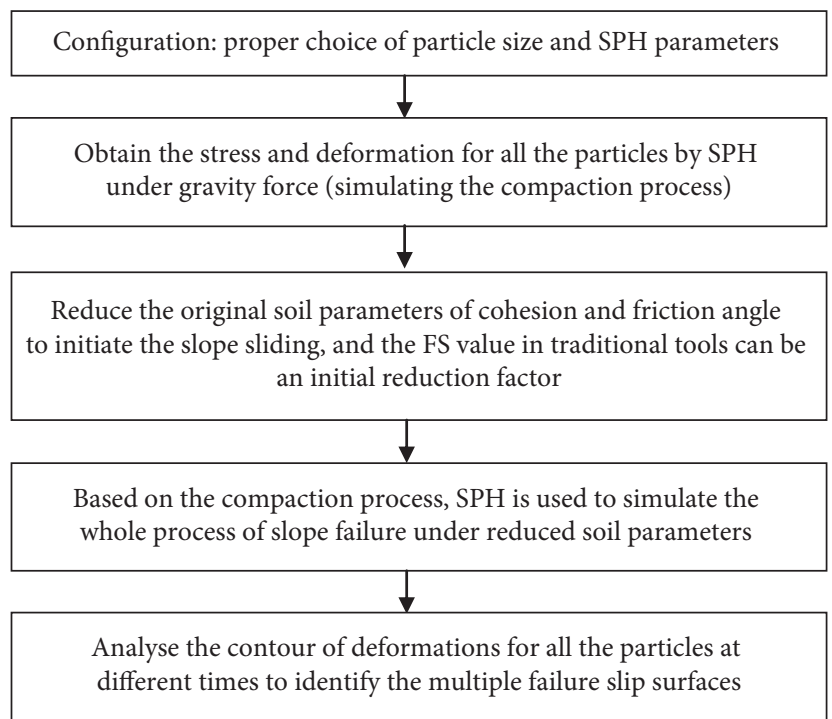

Figure 5: The procedures for the proposed SPH-based approach to locate multiple failure slip surfaces.

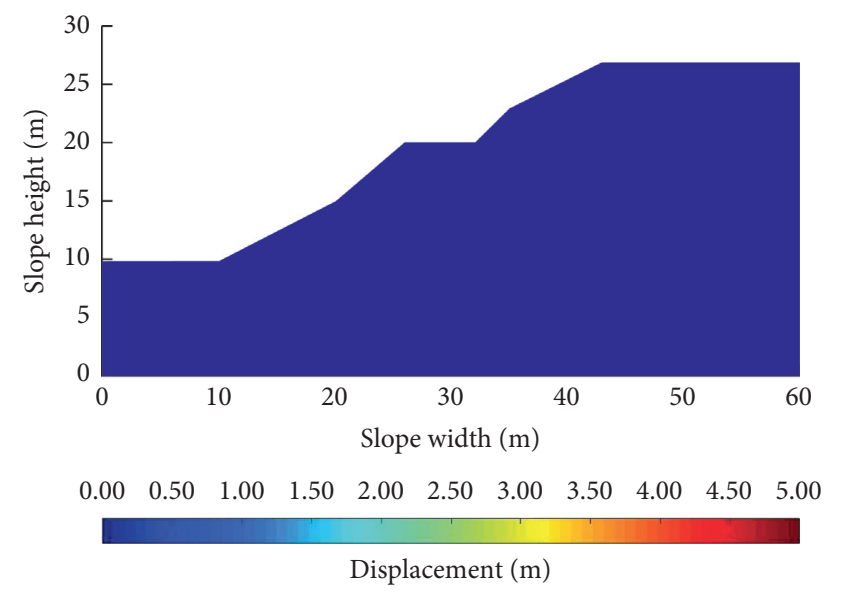

FIGURE 6: Contour plot of deformation for slope particles at steady state. slope to the toe of the slope. The smaller failure zone exhibits a dramatic increase in deformation, and it is the first failure slip surface. A smaller failure zone at the slope top arises in Figure $7(\mathrm{c})$, flows in Figures $7(\mathrm{~d})$ and $7(\mathrm{e})$, and finally emerges into the larger failure zone in Figure 7(f). After $6 \mathrm{~s}$ of simulation, the slope reaches a steady state, and the first failure slip surface at the middle slope shows the largest deformation value, as shown in Figure 7(f). To compare the results in SPH with those in LEM, multiple failure slip surfaces (red dashed lines) with the contour deformation at different simulation times using undeformed particle deposition are shown in Figure 8. The multiple failure slip surfaces obtained by Cheng et al. [10] correspond well to the deformation contour in SPH. Therefore, the multiple sources of failure slip surfaces in slope stability are equivalent to the multiple sources of initiation of slope failure. SPH provides a direct and systematic tool for identifying the failure slip surfaces. Compared with LEM, the deformation of the slope is clearly captured. In fact, very large 


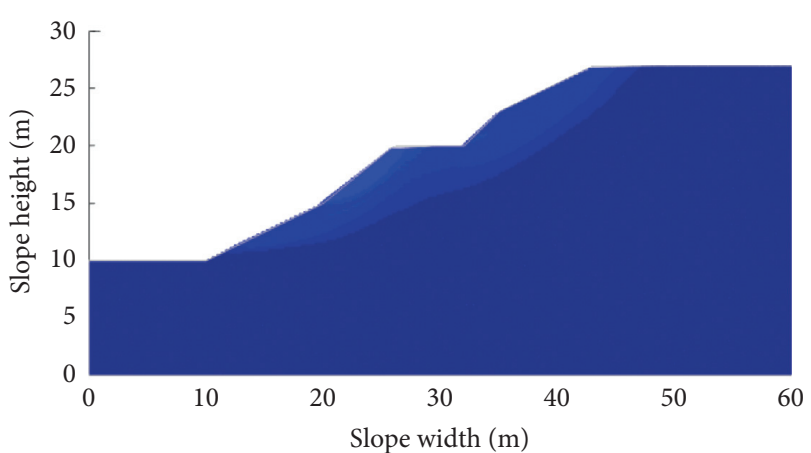

$\begin{array}{lllllllllll}0.00 & 0.50 & 1.00 & 1.50 & 2.00 & 2.50 & 3.00 & 3.50 & 4.00 & 4.50 & 5.00\end{array}$

Displacement $(\mathrm{m})$

(a)

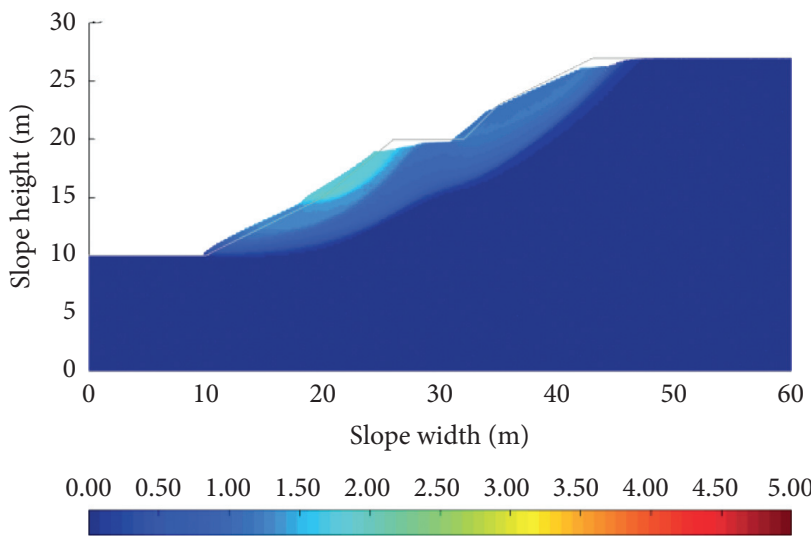

Displacement $(\mathrm{m})$

(c)

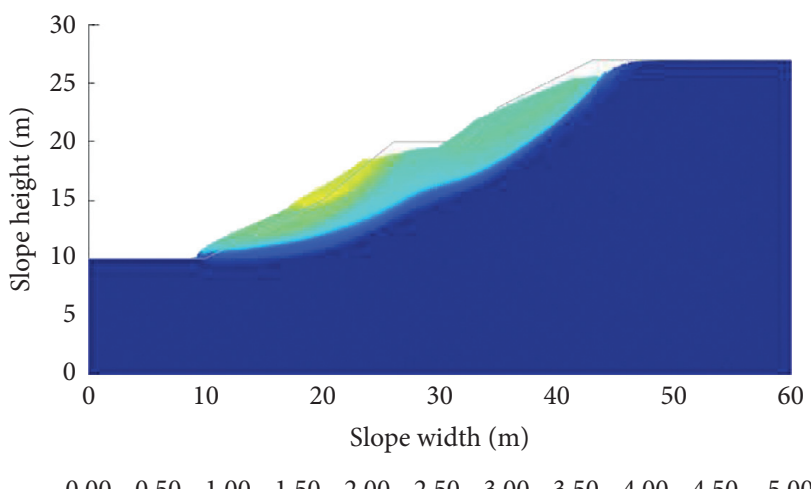

Displacement (m)

(e)

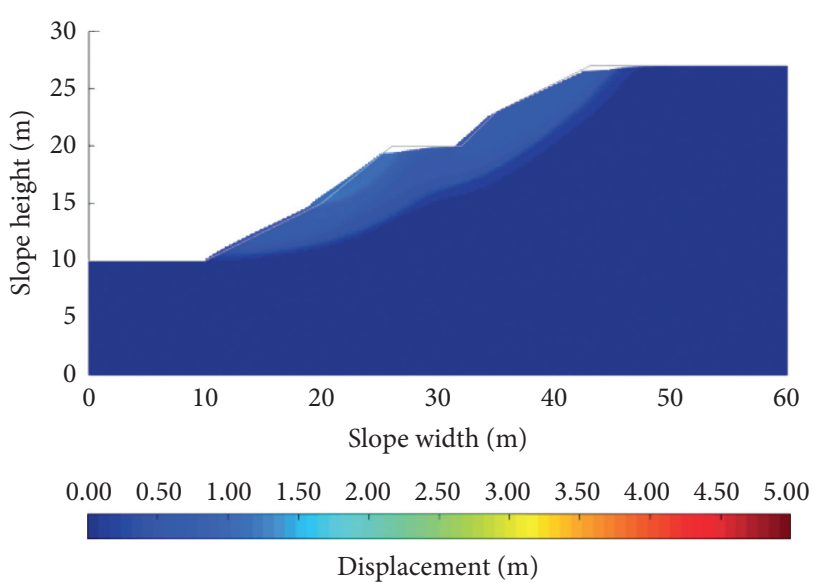

(b)

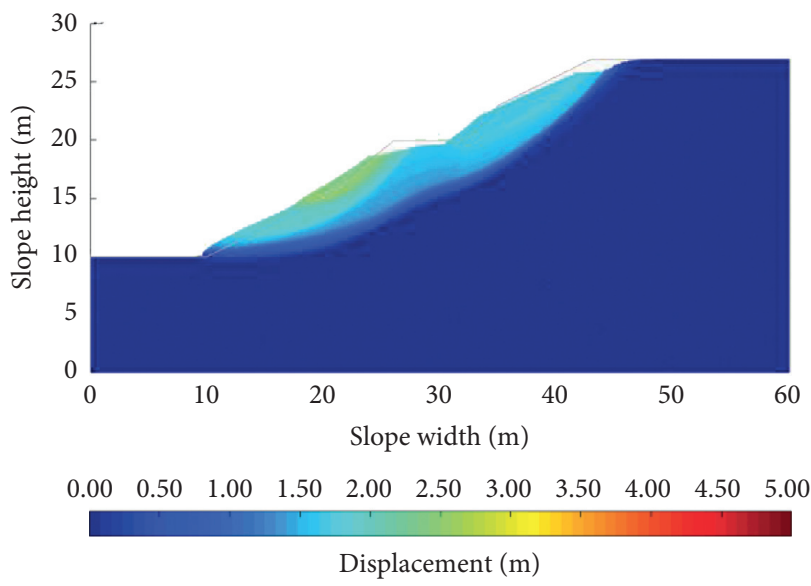

(d)

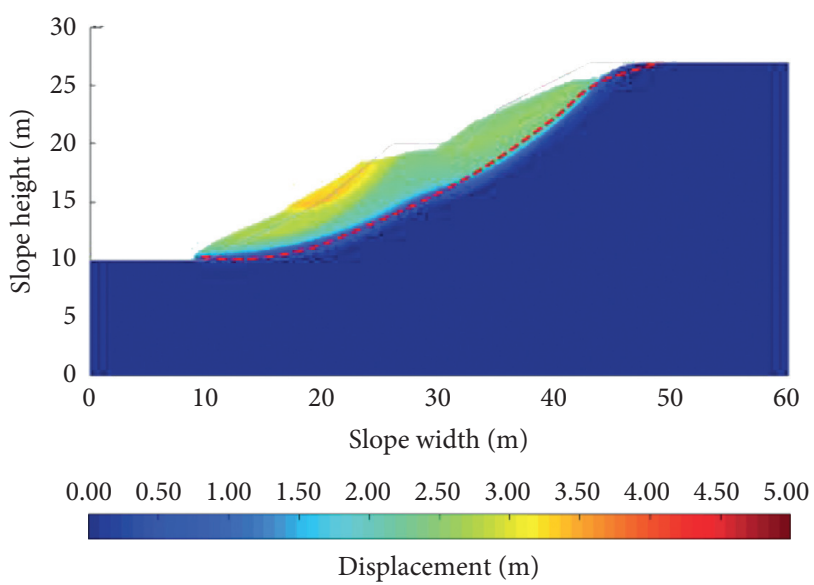

(f)

Figure 7: Contour plot of deformation for slope particles at different times: (a) $t=1 \mathrm{~s}$; (b) $t=2 \mathrm{~s}$; (c) $t=3 \mathrm{~s}$; (d) $t=4 \mathrm{~s}$; (e) $t=5 \mathrm{~s}$; (f) $t=6 \mathrm{~s}$ (the dotted line in (f) represents the result from MPM analysis).

displacement or even flow of the soil mass can be modelled with the SPH analysis.

There is one concern regarding the SPH analysis that is worth consideration. In Figure 7, there is a minor abnormal change in the gradient for the largest critical slip surface near the middle of the slip surface. The lowering of the gradient of the slip surface will induce higher resistance to failure, and such a result appears to be unreasonable. This phenomenon is not reported by the use of LEM or FEM. To check for the acceptability of this slip surface, an in-house-developed material point method (MPM) program is used to analyse this problem again with a factor of safety of 1.35 . As shown 


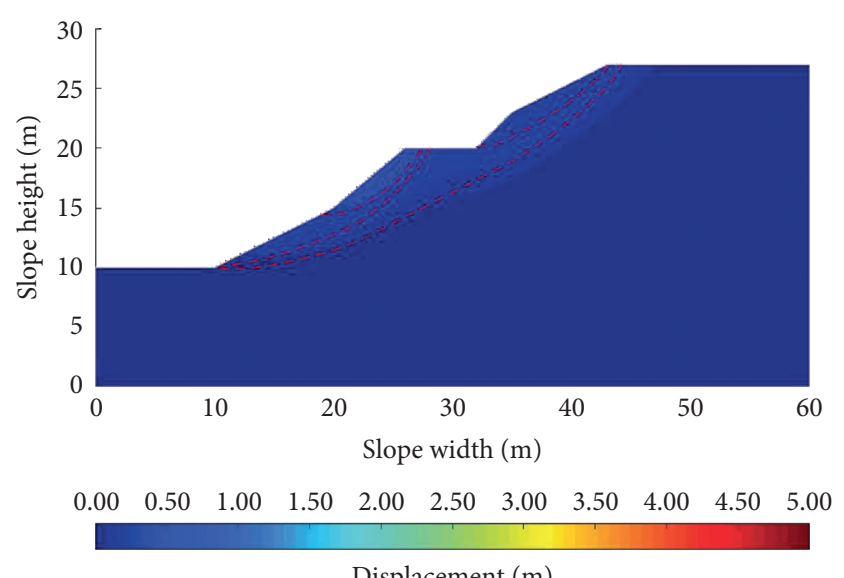

Displacement (m)

(a)

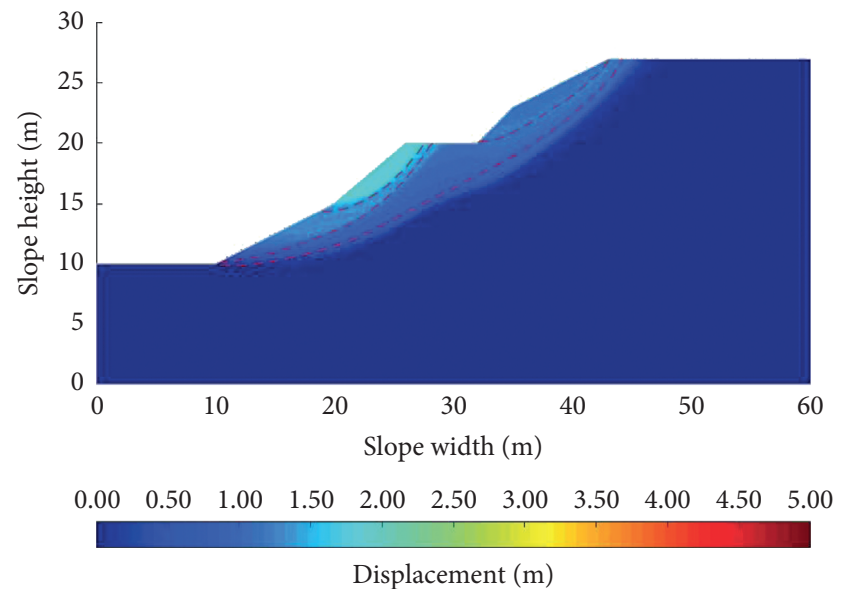

Displacement (m)

(c)

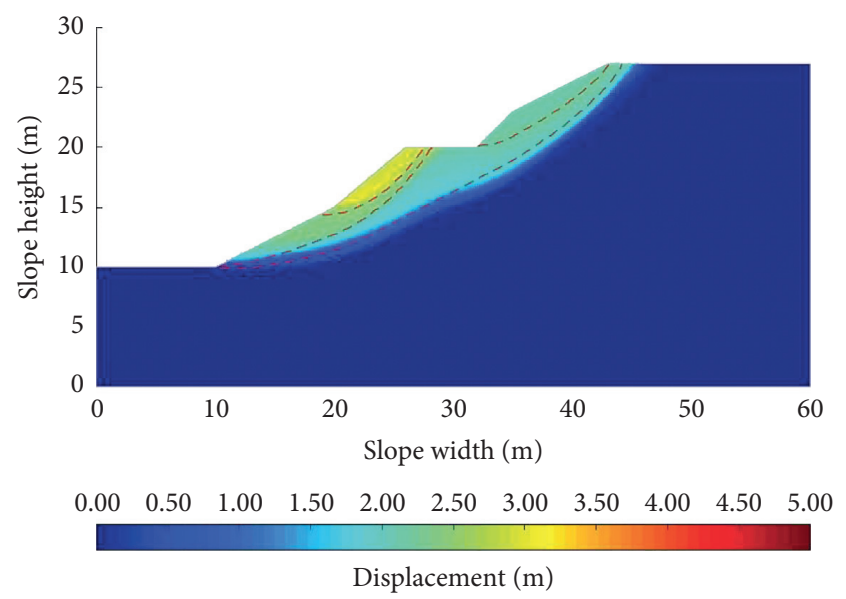

(e)

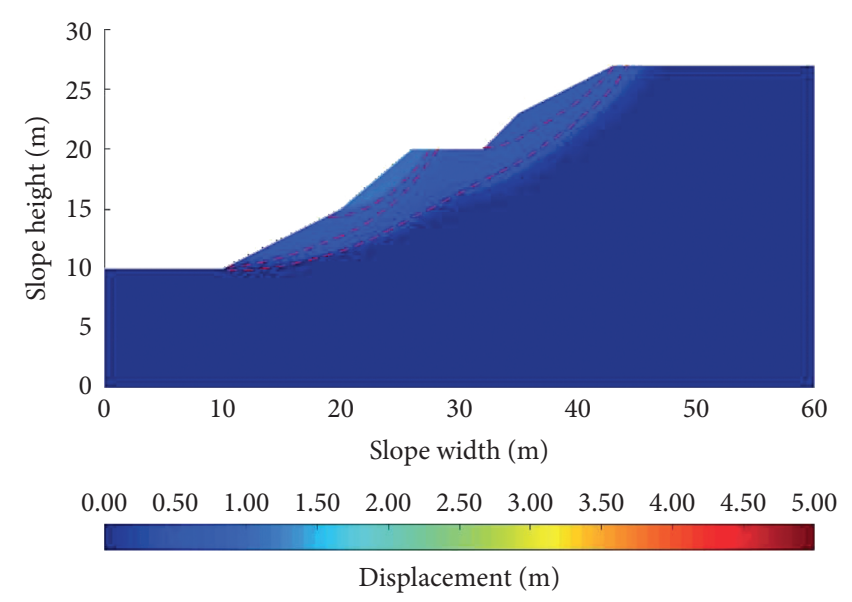

(b)

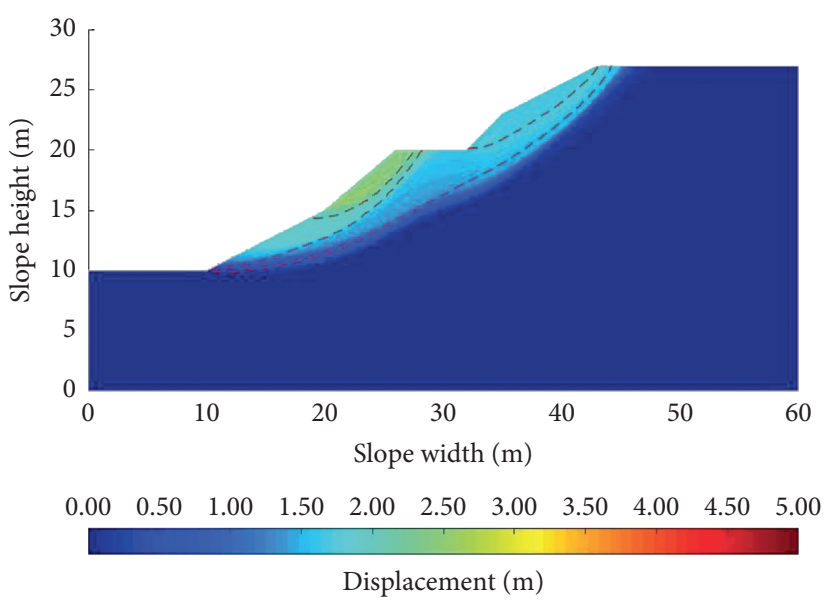

(d)

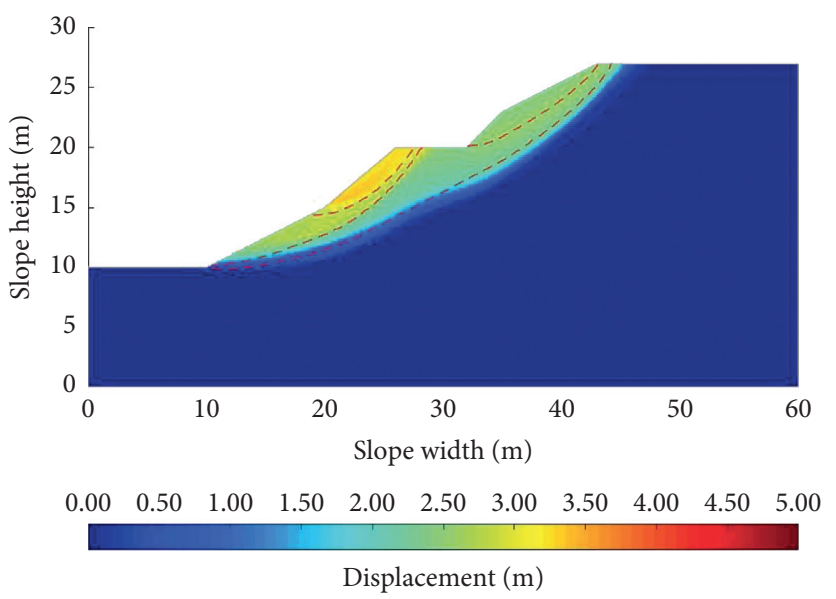

(f)

FIGURE 8: Comparison of multiple failure slip surfaces between SPH (at different times) and LEM [10]: (a) $t=1 \mathrm{~s}$; (b) $t=2 \mathrm{~s}$; (c) $t=3 \mathrm{~s}$; (d) $t=4 \mathrm{~s}$; (e) $t=5 \mathrm{~s}$; (f) $t=6 \mathrm{~s}$.

in Figure 7(f), a smooth slip surface, as shown by the dotted line, is obtained by the MPM, which is also similar to the result by LEM. This result has also indicated that SPH can be affected to different extents by various factors such as kernel function, $\theta$, or $M$. The authors do not view such a limitation to be detrimental for SPH, as LEM, FEM, FDM, and MPM are also affected by various factors in the actual applications. The use of SPH can enable the assessment of multiple critical slip surfaces automatically, which is clearly an important contribution to the analysis of slope stability.

Through the comparison with the evaluation of multiple slip surfaces by the LEM, it is illustrated that the SPH has the 
potential in the autodetection of multiple slip surfaces which is not possible for LEM and is usually difficult with the FEM.

\section{Discussion and Conclusions}

The presence of multiple sources of potential failure slip surfaces is important for the proper design of slope reinforcement works, slope risk assessment, and decision making related to preventing slope failure. This phenomenon may not be critical for normal simple slopes but will be more important for staged slopes and slopes with complicated geometry and ground conditions. Traditional numerical tools including LEM, FEM, and FDM are commonly used for the determination of a single critical slip surface. It is cumbersome to determine the multiple slip surfaces in LEM, and the use of FEM and FDM to automatically locate all the potential sources of failure slip surfaces appears to be generally unsuccessful for most of the commercial programs. By combining the SPH method and strength reduction technique, it is demonstrated that SPH can simulate the whole slope failure process and identify all the failure slip surfaces. The failure slip surfaces identified by the proposed method agree fairly well with those obtained by LEM through varying different entrance and exit search domains. Therefore, the SPH algorithm serves as an effective and alternative tool for identifying the multiple sources of potential failure slip surfaces, and it can provide more insights in the detailed analysis of slope stability problems. The application of SPH to the determination of multiple slip surfaces in slope stability problems in the present paper serves as a pilot study in this area for which there is no previous report about such application, and it is expected that as SPH gets mature in the future, there will be more such applications.

At present, the SPH method is still not mature enough for general engineering use. It is time consuming to carry out an SPH analysis, and the postprocessing of the results from $\mathrm{SPH}$ analysis requires a certain amount of effort. The authors, however, hold the view that SPH can be applied in slope stability problems in the future, although further research is needed for such applications.

\section{Data Availability}

The data used to support the findings of this study are available from the corresponding author upon request.

\section{Conflicts of Interest}

The authors declare that there are no conflicts of interest regarding the publication of this paper.

\section{Acknowledgments}

This study was supported by the National Natural Science Foundation of China (grant no. 51778313), Special Project Fund of Taishan Scholars of Shandong Province, China (2015-212), and State Key program of National Natural Science Foundation of China (grant no. U1802243). The financial supports are gratefully acknowledged.

\section{References}

[1] A. W. Bishop, "The use of the slip circle in the stability analysis of slopes," Géotechnique, vol. 5, no. 1, pp. 7-17, 1955.

[2] N. R. Morgenstern and V. E. Price, "The analysis of the stability of general slip surfaces," Géotechnique, vol. 15, no. 1, pp. 79-93, 1965.

[3] E. Spencer, "A method of analysis of the stability of embankments assuming parallel inter-slice forces," Géotechnique, vol. 17, no. 1, pp. 11-26, 1967.

[4] N. Janbu, Slope Stability Computations Embankment Dam engineering the Casagrande Volume, Wiley and Sons, New York, NY, USA, 1973.

[5] R. Baker, "Determination of the critical slip surface in slope stability computations," International Journal for Numerical and Analytical Methods in Geomechanics, vol. 4, no. 4, pp. 333-359, 1980.

[6] Z.-Y. Chen and C.-M. Shao, "Evaluation of minimum factor of safety in slope stability analysis," Canadian Geotechnical Journal, vol. 25, no. 4, pp. 735-748, 1988.

[7] D. V. Griffiths and P. A. Lane, "Slope stability analysis by finite elements," Géotechnique, vol. 49, no. 3, pp. 387-403, 1999.

[8] E. M. Dawson, W. H. Roth, and A. Drescher, "Slope stability analysis by strength reduction," Géotechnique, vol. 49, no. 6, pp. 835-840, 1999.

[9] Y. M. Cheng, "Location of critical failure surface and some further studies on slope stability analysis," Computers and Geotechnics, vol. 30, no. 3, pp. 255-267, 2003.

[10] Y. M. Cheng, T. Lansivaara, and W. B. Wei, "Two-dimensional slope stability analysis by limit equilibrium and strength reduction methods," Computers and Geotechnics, vol. 34, no. 3, pp. 137-150, 2007.

[11] H. Zheng, G. Sun, and D. Liu, "A practical procedure for searching critical slip surfaces of slopes based on the strength reduction technique," Computers and Geotechnics, vol. 36, no. 1-2, pp. 1-5, 2009.

[12] W. B. Wei, Y. M. Cheng, and L. Li, “Three-dimensional slope failure analysis by the strength reduction and limit equilibrium methods," Computers and Geotechnics, vol. 36, no. 1-2, pp. 70-80, 2009.

[13] L. Li, G.-m. Yu, Z.-y. Chen, and X.-s. Chu, "Discontinuous flying particle swarm optimization algorithm and its application to slope stability analysis," Journal of Central South University of Technology, vol. 17, no. 4, pp. 852-856, 2010a.

[14] Y.-C. Li, Y.-M. Chen, T. L. T. Zhan, D.-S. Ling, and P. J. Cleall, "An efficient approach for locating the critical slip surface in slope stability analyses using a real-coded genetic algorithm," Canadian Geotechnical Journal, vol. 47, no. 7, pp. 806-820, 2010 b.

[15] L. Li and X.-s. Chu, "An improved particle swarm optimization algorithm with harmony strategy for the location of critical slip surface of slopes," China Ocean Engineering, vol. 25, no. 2, pp. 357-364, 2011.

[16] L. Li, Y. M. Cheng, and X.-s. Chu, "A new approach to the determination of the critical slip surfaces of slopes," China Ocean Engineering, vol. 27, no. 1, pp. 51-64, 2013.

[17] L. Li, Y. Wang, Z. Cao, and X. Chu, "Risk de-aggregation and system reliability analysis of slope stability using representative slip surfaces," Computers and Geotechnics, vol. 53, pp. 95-105, 2013.

[18] T. Bai, T. Qiu, X. Huang, and C. Li, "Locating global critical slip surface using the morgenstern-price method and optimization technique," International Journal of Geomechanics, vol. 14, no. 2, pp. 319-325, 2014. 
[19] Y. Tu, X. Liu, Z. Zhong, and Y. Li, "New criteria for defining slope failure using the strength reduction method," Engineering Geology, vol. 212, pp. 63-71, 2016.

[20] L. Li and X. Chu, "Failure mechanism and factor of safety for spatially variable undrained soil slope," Advances in Civil Engineering, vol. 2019, Article ID 8575439, 17 pages, 2019.

[21] K. Krabbenhoft, A. V. Lyamin, M. Hjiaj, and S. W. Sloan, "A new discontinuous upper bound limit analysis formulation," International Journal for Numerical Methods in Engineering, vol. 63, no. 7, pp. 1069-1088, 2005.

[22] C. C. Smith and M. Gilbert, "Identification of rotational failure mechanisms in cohesive media using discontinuity layout optimisation," Géotechnique, vol. 63, no. 14, pp. 1194-1208, 2013.

[23] Y. M. Cheng, Slope Stability and Reliability Analysis, Nova Publishing, Hauppauge, NY, USA, 2018.

[24] C. Reale, J. Xue, Z. Pan, and K. Gavin, "Deterministic and probabilistic multi-modal analysis of slope stability," Computers and Geotechnics, vol. 66, pp. 172-179, 2015.

[25] L. Li and X. Chu, "Locating the multiple failure surfaces for slope stability using monte carlo technique," Geotechnical and Geological Engineering, vol. 34, no. 5, pp. 1475-1486, 2016.

[26] L. B. Lucy, "A numerical approach to the testing of the fission hypothesis," The Astronomical Journal, vol. 82, no. 12, pp. 1013-1024, 1977.

[27] R. A. Gingold and J. J. Monaghan, "Smoothed particle hydrodynamics: theory and application to non-spherical stars," Monthly Notices of the Royal Astronomical Society, vol. 181, no. 3, pp. 375-389, 1977.

[28] J. J. Monaghan, "Smoothed particle hydrodynamics," Annual Review of Astronomy and Astrophysics, vol. 30, no. 1, pp. 543-574, 1992.

[29] J. J. Monaghan, "SPH without a tensile instability," Journal of Computational Physics, vol. 159, no. 2, pp. 290-311, 2000.

[30] G. R. Liu and M. B. Liu, Smoothed Particle Hydrodynamics: A Mesh Free Particle Method, World Scientific, Singapore, 2004.

[31] H. H. Bui, R. Fukagawa, K. Sako, and Y. Okumura, "Earthquake induced slope failure simulation by SPH," in Proceedings of the Fifth International Conference on Recent Advances in Geotechnical Earthquake Engineering and Soil Dynamics and Symposium in Honour of Professor I. M. Idriss, pp. 1-9, San Diego, CA, USA, 2010.

[32] H. H. Bui, R. Fukagawa, K. Sako, and J. C. Wells, "Slope stability analysis and discontinuous slope failure simulation by elasto-plastic smoothed particle hydrodynamics (SPH)," Géotechnique, vol. 61, no. 7, pp. 565-574, 2011.

[33] H. H. Bui and R. Fukagawa, "An improved SPH method for saturated soils and its application to investigate the mechanisms of embankment failure: case of hydrostatic pore-water pressure," International Journal for Numerical and Analytical Methods in Geomechanics, vol. 37, no. 1, pp. 31-50, 2013.

[34] Y. An, Q. Wu, C. Shi, and Q. Liu, "Three-dimensional smoothed-particle hydrodynamics simulation of deformation characteristics in slope failure," Géotechnique, vol. 66, no. 8, pp. 670-680, 2016.

[35] H. Nonoyama, A. Yashima, and S. Moriguchi, "Future evolution of slope stability analysis created by SPH method," in Proceedings of the 18th International Conference on Soil Mechanics and Geotechnical Engineering, pp. 2229-2232, Paris, France, January 2013.

[36] H. Nonoyama, S. Moriguchi, K. Sawada, and A. Yashima, "Slope stability analysis using smoothed particle hydrodynamics (SPH) method," Soils and Foundations, vol. 55, no. 2, pp. $458-470,2015$.
[37] Z. Mao, G. R. Liu, and X. Dong, “A comprehensive study on the parameters setting in smoothed particle hydrodynamics (SPH) method applied to hydrodynamics problems," Computers and Geotechnics, vol. 92, pp. 77-95, 2017.

[38] L. Li and Y. Wang, "Identification of failure slip surfaces for landslide risk assessment using smoothed particle hydrodynamics," Georisk: Assessment and Management of Risk for Engineered Systems and Geohazards, vol. 14, no. 2, pp. 91-111, 2020.

[39] L. Li, Y. Wang, L. M. Zhang, C. Choi, and C. W. W. Ng, "Evaluation of critical slip surface in limit equilibrium analysis of slope stability by smoothed particle hydrodynamics," International Journal of Geomechanics, vol. 19, no. 5, Article ID 04019032, 2019.

[40] L. Li, X. Chu, and G. Yu, "Cohesive slope failure analysis using methods combining smoothed particle hydrodynamics and response surface function," Engineering Computations, vol. 37, no. 3, pp. 1093-1108, 2020.

[41] X. Chu, L. Li, and Y. M. Cheng, "Risk assessment of slope failure using assumption of maximum area of sliding mass and factor of safety equal to unit," Advances in Civil Engineering, vol. 2019, Article ID 6268079, 11 pages, 2019.

[42] X. Chu, L. Li, G. Yu, and C. Yuan, "Comparison of slope failure areas between limit equilibrium method and smoothed particle hydrodynamics," European Journal of Environmental and Civil Engineering, pp. 1-17, 2019.

[43] H. H. Bui, R. Fukagawa, K. Sako, and S. Ohno, "Lagrangian meshfree particles method (SPH) for large deformation and failure flows of geomaterial using elastic-plastic soil constitutive model," International Journal for Numerical and Analytical Methods in Geomechanics, vol. 32, no. 12, pp. 1537-1570, 2008.

[44] W. L. Wong, "Slope stability analysis by meshless method in Shum Wan Road landslide," Degree report, Hong Kong Polytechnic University, Hung Hom, Hong Kong, 2018.

[45] H. Chen and C. F. Lee, "Numerical simulation of debris flows," Canadian Geotechnical Journal, vol. 37, no. 1, pp. 146-160, 2000. 